\title{
Über Bedingungen wissenschaftlicher Leistung in der europäischen Kultur der Neuzeit
}

\author{
Von Eduard Fueter, Wädenswil
}

\section{Einleitung}

In der Geschichte der Menschheit bedeuten Ausbildung und Entfaltung wissenschaftlicher Forschung im Abendland einen einzigartigen Beitrag. Damit hat dieses sich in das goldene Buch der Jahrtausende eingetragen, und dadurch erhält die Wissenschaftshistorie auch ihre besondere Weihe: sie ist die verantwortungsbewußte Wahrerin eines schon vorher vielfältig vorbereiteten Kulturbewußtseins und eines hoffentlich lebendig fortwirkenden Erbes, dessen lautere ursprüngliche Quellen und Kräfte sie immer wieder aufsucht.

Unter den hervorragenden Werken der Vergangenheit in dieser Disziplin ist leider und auffallenderweise eines beinahe in den Schatten der Vergessenheit geraten, das einen Meilenstein bildet, wie jene noch größere Lebensarbeit, die an dieser Stelle gefeiert werden soll.

Es ist dies die 1873 erstmals, 1885 in beträchtlich erweiterter zweiter Auflage vorgelegte «Histoire des sciences et des savants depuis deux siècles, précédée et suivie d'autres études sur les sujets scientifiques, en particulier sur l'hérédité et la sélection dans l'espèce humaine» von Alphonse de CANDoLle, erschienen bei H. Georg, Genève-Bâle.

Die hohe Bedeutung dieser Schrift wurde zwar sogleich erkannt. Das trefflichste Lob spendete ihr nach dem Tode des Verfassers der als Herausgeber der «Klassiker der exakten Wissenschaften» sehr verdiente WiLHELm Ostwald. Er gab als zweiten Band seiner Sammlung «Große Männer. Studien zur Biologie des Genies» den Band des Genfers in deutscher Übersetzung (Leipzig 1911) neu heraus und versah sie mit einer höchst anerkennenden Einführung. Im besondern erklärte er, daß DE CANdolle mit seiner Studie «das Fundamentalwerk einer neuen Wissenschaft» ${ }^{1}$ geschaf-

\footnotetext{
${ }^{1}$ Deutsche Ausgabe (1911), S. V. - In der Folge wird stets ohne weiteren Zusatz nach dieser Ausgabe zitiert. Die anscheinend von Ostwald selbst besorgte Übersetzung ist allerdings manchmal schwerfällig und gelegentlich gewaltsam, indem Ostwald den Genfer gerne in seinem Sinne interpretierte. Um der äußern, sprachlichen Einheit willen - die sonst dauernd unterbrochen werden müßte - stützen wir uns aber darauf; in gewissen Fällen ist auf das Original verwiesen, soweit dies notwendig schien.
} 
fen habe, nämlich die «Wissenschaft von den führenden Menschen innerhalb des Kulturkreises» oder die «Geniologie».

Möglicherweise ist es aber gerade diese betonte Anerkennung durch Ostwald gewesen, die DE CANDolles allgemeinem Ansehen in der nächsten Generation mehr geschadet als genützt hat. So unbestritten manche Leistungen Ostwalds sind - wie etwa seine Farbenlehre oder sein Verdünnungsgesetz -, so haben die Art seiner monistischen Überzeugungen, vor allem aber seine Energetischen Grundlagen der Kulturwissenschaft (1922) das Kopfschütteln oder die Ablehnung sowohl der Naturforscher wie der Kulturhistoriker hervorgerufen. In den Bann ihrer Verurteilung ist wahrscheinlich auch DE CANDOLLE geraten. Und nichts hätte diese noch verstärken können als die von OsTwALD geschaffene, abschreckende Etikette: «Geniologie!»

Aber damit wurde einem ausgezeichneten Gelehrten und Werk sowie dem Sproß einer berühmten Familie Unrecht getan. De CANdolle hat mit OstwaLDS philosophischen und «kulturenergetischen» ${ }^{2}$ Überzeugungen nichts zu tun.

Fataler als die Minderung seines Ansehens war noch, daß dE CANDolles bedeutende Untersuchungen kaum ernsthafte Nachfolge fanden (sofern man von gewissen amerikanischen soziologischen oder einigen eher oberflächlichen europäischen kulturgeschichtlichen Analysen ohne gründliche Kenntnisse der Wissenschaftsgeschichte absieht).

So mag es angezeigt sein, einige der sorgfältig überdachten Gedankengänge des Genfers in Erinnerung zu rufen, gelegentlich kritisch zu prüfen oder durch Gegenüberstellung zu fast gleichzeitigen Äußerungen JАсов BURсKHARDTS zu beleuchten. Damit sind freilich nur Hinweise gegeben. Das Thema ist unerschöpflich und tief, so daß nur ein großangelegter Essay eine allseitige Abklärung bringen könnte. Doch würde dieser den hier gegebenen Rahmen und Zweck weit übersteigen.

2 Es kann kaum ein Zweifel sein, daß die «Kulturenergetik» ein untauglicher Versuch war. Aber das Urteil ist doch wohl zu ablehnend oder hart ausgefallen. In seiner Sprache hat OstwaLD manchmal nur eine Vorstellung gehegt, die auch dem «élan vital» von BERGson zugrunde liegt. Dieser Begriff aber wurde ein - übrigens noch keineswegs granitner «Eckpfeiler» von ARNoLD J. ToynBeEs monumentalem kulturgeschichtlichem Torso: A Study of History (London 1933-1939). - Es besteht übrigens Hoffnung, daß dieses Pionierwerk analysierender und vergleichender Weltgeschichte des Aufstieges, Stillstandes und Niederganges der «civilizations» noch im vorgesehenen Umfang abgeschlossen werden kann. 
Um das Thema möglichst fruchtbar zu gestalten, wird vor allem ein Aspekt herausgegriffen: die individuellen Bedingungen wissenschaftlicher Größe, wie sie dem Wissenschaftshistoriker im 17. und 18. Jahrhundert entgegentreten und wie sie DE CANDOLLE in einem Hauptstück seines Werkes zu beschreiben trachtete. Dagegen werden die politischen, religiösen, wirtschaftlichen, sozialen und geistesgeschichtlichen Voraussetzungen eher nur gestreift. Es erscheint dies berechtigt, da DE CANDOLLE diese oft verkannte, bzw. die Forschungen der letzten achtzig Jahre hier die vielseitigsten Erkenntnisse brachten.

Wie in vielen ähnlichen Fällen haben eigene Erfahrungen und Persönlichkeit des Autors die Darstellung stark beeinflußt. Sie seien daher kurz geschildert.

\section{Lebensgang und Persönlichkeit Alphonse de Candolles}

Die DE CANDOlle waren französische Réfugiés, die 1558 in Genf ansässig wurden. Sie zählen damit zu jenen zahlreichen Geschlechtern aus dem romanischen Europa, denen die Schweiz und besonders Genf eine ungewöhnliche Zahl hervorragender Forscher verdankt. Durch sie wurde die «res publica Helvetica eruditorum» zu einem Sammelbecken bester Talente abendländischer Kultur. Das unwiderleglich nachzuweisen, war ein Hauptanliegen Alphonse de CANdolles.

Alphonse wurde am 27. Oktober 1806 in Paris dem damals hochangesehenen Botaniker Augustin-Pyramus de Candolle - einem Forscher, dem die systematische Botanik zu einem guten Teil ihre heutige Gestalt verdankt - und seiner Mutter ToRRAS, ebenfalls einer Genferin, geboren. Sein Vater wurde 1807 Professor der Botanik in Montpellier, wo Alphonse daher seine Jugendjahre verbrachte. 1814 siedelte die Familie wieder nach Genf über.

Aus wirtschaftlichen Gründen, d. h. um die Existenz besser als durch ein Studium der reinen Wissenschaften sichern zu können, wurde Alphonse veranlaßt, Jurisprudenz zu studieren. Mit dreiundzwanzig Jahren schloß er dieses Studium mit einer Dissertation über die Begnadigung ab. Die Arbeit war juristisch und menschlich so wertvoll, daß diesem Erstling eine zweite Auflage zuteil wurde.

Das väterliche Interesse hatte ihn aber früh und gründlich gepackt. Wenige Jahre nach dem akademischen Abschluß schrieb er eine Monographie über die Campanulaceen, die durch «ihre sorgfältige Berücksichtigung der geographischen Verbreitung dieses Geschlechts bereits eine we- 
sentliche Richtung seiner späteren hervorragenden Tätigkeit als Pflanzengeograph kennzeichnete». Er wurde daher bald zum Honorarprofessor der Botanik an der Genfer Akademie ernannt. Dabei ging er seinem Vater beim Unterricht wie bei den Vorbereitungen des Prodromus systematis naturalis regni vegetabilis tüchtig an die Hand. In der Fortführung der von seinem Vater unter Mitarbeit zahlreicher anderer Systematiker 1824-1839 herausgegebenen sieben Bände, denen Alphonse bis 1873 zehn weitere beifügte, sollte er zu besonderem Ruhme kommen und sein fachliches Können seinem Vater ebenbürtig an die Seite stellen ${ }^{3}$. Für die in der Schweiz so typische und gerade auch von ihm hervorgehobene «kollektive Leistung» der Gelehrtenfamilie war er also ebenso ein neuer Beweis wie ein unmittelbarer Beobachter. Aus diesen Arbeiten wuchsen seine Lois de la nomenclature botanique (1867, deutsch 1868) heraus, welche vom Internationalen Botanischen Kongreß in Paris 1867 als allgemeingültig anerkannt wurden.

Offensichtlich beschäftigte ihn während dieser Jahre ständig der Gedanke, ob es nicht ähnlich wie bei den Pflanzen besonders günstige «Wachstumsbedingungen» für die Menschen, und zwar insbesondere für den wissenschaftlichen Geist gebe. Da erschien Darwins erstes Werk über die Variation und Selektion, welche DE CANDolle durch eine botanische Sonderarbeit $^{5}$, die mehrfach nachgedruckt wurde, zu prüfen suchte, aber auch auf Erscheinungen der Menschheit ausdehnte. Candolle schloß darin auf die außerordentliche Langsamkeit der Selektion und eine durchaus untergeordnete Wirkung auf die meisten Spezies. Insbesondere erklärte er: «Unter diesem Gesichtspunkt sind die menschlichen Rassen lehrreich. Sicherlich haben die alten Hebräer, Griechen und Römer und die Menschen der weißen Rasse intensiv gekämpft, sowohl einzeln wie kollektiv. Die Schwächsten sowohl in physischer wie intellektueller Beziehung sind dabei stets im Nachteil geblieben und die physisch oder moralisch Stärksten haben stets gesiegt. Und dennoch kann man nicht sagen, daß zwischen den Alten und den Modernen ein großer Unterschied besteht, weder bezüglich der

${ }^{3}$ Er würdigte zudem in seiner Géographie botanique raisonnée (2 Bände, 1855) erstmals die geologischen Ursachen der heutigen Pflanzenverbreitung eingehend. Den Kulturpflanzen galt sein Werk Origine des plantes cultivées (2. Auflage 1883, deutsch 1884).

${ }^{4}$ Daraus ist eines der besten Kapitel des Werkes und einige der bestimmtesten Überzeugungen entsprungen, z.B.: «Man kann im voraus eins gegen zehn wetten, daß aus Familien, in welchen man die Trägheit oder den Luxus allein als etwas Rühmliches betrachtet, kein Fortschritt der Wissenschaft ergebener Gelehrter hervorgehen wird» (S. 269).

${ }^{5}$ Archives des sciences naturelles, Oktober 1862. 
Intelligenz noch der physischen Schönheit, der Stärke, der Gesundheit. Man kann sogar annehmen, daß er Null ist, denn die einen schätzen den Unterschied in dem einen Sinne, die anderen im entgegengesetzten» ${ }^{6}$.

Nachdem DE Gandolle diese Gedankenreihe fortgesponnen hatte, erschienen in kurzer Folge die Bücher von Wallace, Contribution to the Theory of Natural Selection; Herbert Spencer, Principles of Biology (2 vol., London 1867), und Sir Francis Galton, Hereditary Genius (London 1869). Sie wurden 1871 von DARwIN im ersten Band seines Buches über den Ursprung des Menschen zusammengefaßt ${ }^{7}$.

Die Wirkung dieser Schriften, insbesonders von Galton, auf de CaNDOLLE war entscheidend. Sie wiesen ihn nachdrücklich auf die Bedeutung und die Probleme der Vererbung hin. Gleichzeitig aber stritten sie in seinem Innern mit seinen historischen Beobachtungen. Sie führten zu dem ebenso interessanten wie gedankenvollen Abschnitt: «Erblichkeit und Verschiedenheit der aufeinanderfolgenden Generationen", worin er mit der grundlegenden Überzeugung begann: «Die Vererbung der physischen, moralischen

${ }^{6}$ Die Kulturgeschichtsschreibung und moderne Historiographie haben im Laufe der letzten hundert Jahre eindeutig gezeigt, daß mit so einfachen Gedankengängen dieses Problem nicht zu lösen ist. Der Wettbewerb der Völker und Kulturen spielt sich in weit differenzierteren Formen ab, wofür TOYNBEE die Formel des «challenge and response» geprägt hat. Aber offensichtlich genügt auch diese noch nicht oder muß noch tiefer gefaßt werden, als bisher versucht wurde. So spielt offensichtlich ein Faktor auch eine bedeutende Rolle, die in ebenso geistvoller wie überzeugender Weise HENRY E. SigerisT in seinem Werke Civilization and Desease aufwies: die Wechselwirkung von Erkrankungen aller Art bei kollektiven oder individuellen Organismen. Mindestens auf dem Gebiete der Kulturentfaltung ist dabei die vereinfachte Selektionstheorie von DARwIN völlig unbrauchbar: zwischen der Leistungsfähigkeit einer Zivilisation und der Anfälligkeit gegenüber gewissen Epidemien besteht kein so simples Verhältnis, daß dekadente Kulturen von Epidemien befallen, schöpferische aber verschont bleiben. So haben z.B. nach medizin-historischen Untersuchungen die Athener unter Perikles, also in einem Blütezeitalter, vor allem wegen der bei ihnen ausgebrochenen «attischen Seuche» im ersten Peleponnesischen Krieg ihre Hegemonie auf dem Festlande verloren; die Folgen auf andere, weniger entwickelte Gemeinwesen war geringer. (Vgl. H.Schlossberger, Kriegsseuchen. Historischer Überblick über ihr Auftreten und ihre Bekämpfung, Jena 1945, S. 2f.) Diese Niederlage führte aber dann zu schweren politischen und schließlich kulturellen Folgen. Unzweifelhaft verdient auch dieses Beispiel noch weitere Ausführungen und kritische Prüfung. Sicher ist stets, daß nicht ein Faktor in der Regel für Aufstieg oder Niedergang von Kulturen ausschlaggebend ist, sondern das Zusammenwirken vieler, oft sehr vieler Kräfte, wobei gleiche Faktoren in veränderter Umwelt unterschiedliche Folgen zeitigen.

7 Seite 21: De Candolle hatte schon vorher einen Essay über Vererbung und Selektion beim Menschen geschrieben. 
und intellektuellen Eigenschaften bei den Menschen hat oft zu falschen und übertriebenen Ansichten Anlaß gegeben.» Damit wies er die damals weitverbreitete Ansicht zurück, daß die Probleme der menschlichen Geschichte genau wie jene der Naturforschung (vor allem der Biologie, der Zoologie oder der Botanik) behandelt werden können. Zugleich aber suchte er die ihm in der Pflanzenkunde so vertrauten und erfolgversprechenden Methoden scharfer Beobachtung und der eben von QUETELET weiterentwickelten Statistik auf historische Erscheinungen anzuwenden. Daß er dabei im ganzen so richtig vorging, verdankte er wahrscheinlich seinem juristischen Studium, welches ihn für die Eigenart von Sozialphänomenen geschult hatte. Diese Methode im einzelnen zu schildern, kann hier nicht der Ort sein. Aber sie war eine bedeutende, in ihrer Originalität bisher nicht gewürdigte Pioniertat. Sie hat ihn so weitreichende Probleme wie die Fragen nach den Ursachen der Häufung großer Forscher im 18. und 19. Jahrhundert bei den einzelnen Individuen aufgreifen und nach objektiven Kriterien ihrer Wertung suchen lassen. - Obgleich auch er, wie nicht anders möglich, die Probleme vereinfachte und die Methode zahlreiche Schwächen aufwies, so zeigte sie sich doch im ganzen als fruchtbar und förderlich. Der Hauptmangel ist unzweifelhaft, daß er den geistesgeschichtlichen Voraussetzungen keine oder völlig ungenügende Aufmerksamkeit schenkte. So kommt es, daß alle seine Schlüsse nur richtig sind, wenn eine bestimmte geistesgeschichtliche Konstellation besteht, die er aber nicht beschrieb oder analysierte. Es war in erster Linie Wilhelm Dilthey vorbehalten, hier die bahnbrechenden Untersuchungen vorzunehmen in seiner Einleitung in die Geisteswissenschaften (erstmals erschienen 1883) und in seinen unvergänglichen Abhandlungen Weltanschauung und Analyse des Menschen seit Renaissance und Reformation (1891-1904 als Essays veröffentlicht).

De Candolles Werk wurde sogleich nach Erscheinen sehr gut aufgenommen und erlebte, wie erwähnt, eine zweite, stark erweiterte Auflage. Insbesonders wurde er mit der Mitgliedschaft vieler angesehener Akademien ausgezeichnet, auf die hinzuweisen er auf dem Titelblatt der 2. Auflage seiner Histoire nicht unterließ. Allgemein betrauert, starb er in Genf am 4. April 1893. Sein Sohn Casimir Pyrame, gleichfalls Botaniker, sollte den Vater und die Familie nochmals zu europäischer Geltung bringen. 


\section{III. «Verschiedene Ursachen, welche die Anzahl, die Richtung und den Erfolg der Menschen bestimmen, welche den Fortschritt der Wissenschaft bewirken»}

Nachdem de Candolle in zwei Abschnitten seine auf die Geschichte angewandte Beobachtungsmethode geschildert, die «Statistik als reguläres Beobachtungsverfahren» bei Sozialphänomenen begründet, den Einfluß der Vererbung und der Auswahl auf die Entwicklung des Menschengeschlechts (mit einem bemerkenswerten Kapitel über die Zukunft desselben ${ }^{8}$ ) angeschlossen und «Über das notwendige Alternieren in der Intensität der Krankheit und die Wirksamkeit der Gegenmittel, z. B. bei der Schutzpockenimpfung» Wesentliches geschrieben hatte, fügte er das Hauptstück ein: «Geschichte der Wissenschaft und der Forscher seit zwei Jahrhunderten, gemäß dem Urteil der wichtigsten Akademien und wissenschaftlichen Gesellschaften.» In dieser Darstellung kommt dem vierten Teil: «Analyse der Tatsachen und Untersuchung der Ursachen, welche die Entwicklung der Wissenschaft fördern oder hemmen», grundlegende Bedeutung zu. Gesamthaft darf er klassische Geltung beanspruchen, auch wenn er im einzelnen unrichtig oder stark überholt ist. Er bespricht darin u. a. das Verhältnis der Mathematiker zu den Naturforschern seit zwei Jahrhunderten, die Rolle der Spezialisierung und jene der Frauen für den wissenschaftlichen Fortschritt, dann die - aus aristokratischer Warte geschriebene - soziologische Betrachtung über die Schichten, aus denen die Gelehrten stammen, den Anteil der einzelnen Nationen an der modernen Entwicklung der Forschung usf. Das Kernstück hier bildet der 5. Paragraph mit der oben gegebenen Überschrift. Diesem soll nun besondere Aufmerksamkeit gelten'.

Bevor DE CANDOLle auf Einzelheiten eingehen will, definiert er klar, was er unter einem Forscher versteht: «Der Probierstein zur Erkenntnis eines solchen Forschers ist nicht ein Examen, sondern daß er nach wirklichen Dingen ${ }^{10}$ begierig ist, die nicht oder schlecht bekannt sind, und daß er die Wahrheit um ihrer selbst willen liebt, ohne sich um die Meinung anderer, um persönliche Vorteile oder mögliche Folgen zu kümmern. Es handelt sich um eine freiwillige selbstlose Arbeit, für welche ein gewisses $\mathrm{Ma} ß$ von Ausdauer und Fähigkeit notwendig ist.»

${ }^{8}$ Diese Betrachtung ist im ganzen dem Zeitdenken gemäß optimistisch, bietet aber auch originelle Aspekte.

${ }^{9}$ Die sprachliche Schwerfälligkeit mangelt dem französischen Original.

${ }^{10}$ Im Original steht «choses réelles», was einen weitern Sinn hat. 
Zahlreiche Ursachen wirken auf diese moralischen und psychologischen Bedingungen ein. Er unterscheidet darin drei Klassen:

«1. Ursachen, die vor der Geburt liegen, wie ererbte Fähigkeiten, Fehler und Neigungen bei den Eltern und Vorfahren.

2. Variationen, d. h. die Erscheinung neuer Charaktere, die in der Familie noch nicht vorkamen.

3. Einflüsse nach der Geburt, wie Erziehung, Beispiel, Ratschläge, persönliche Erfahrungen, Nachdenken infolge der Studien, Umstände der Umgebung, öffentliche Meinung, Institutionen des Landes.»

Diese Darstellung entsprach einer Entgegnung und Kritik an dem im übrigen hochgeschätzten GALton. Denn dieser hatte vornehmlich oder ausschließlich die Erblichkeit des «Genies» behauptet. De CANdolle aber wollte die Faktoren der äußern und seelischen Umwelt, des Milieus in seiner weitesten Bedeutung, mitberücksichtigen. «Ich darf mir schmeicheln, daß ich so mehr in das Innere der Frage eingedrungen bin.» ${ }^{11}$ Dabei unternahm er den klugen Schritt, sich in der Auswahl seiner Studien an Gelehrten auf die letzten beiden Jahrhunderte zu beschränken, während GALTON - in gelegentlich etwas dilettantischer Weise ${ }^{12}$ - an 65 ausgewählten Persönlichkeiten seit dem Altertum exemplifiziert hatte, deren Lebensdaten oft schwer bestimmbar waren. Außerdem besaß dE CANdolle den methodischen Vorteil, daß sein «Beobachtungsmaterial» sich auf einige hundert Exemplare der Species «homo sapiens eruditissimus Europensis» ${ }^{13}$ erstreckte. Das zu erreichen, entsprach einer Notwendigkeit für DE CANdolle: er wollte womöglich statistisch vorgehen, und wußte zudem aus der Botanik, wie die Untersuchung an einzelnen Pflanzen gleicher Art, aber verschiedenen Standorts, gefährlich ist.

In der Folge bemerkte dE CANDolle allerdings, daß auch seine Beobachtungsreihe zu klein ist ${ }^{14}$, um Statistiken zu erstellen. Daher ergab sich die

${ }^{11}$ S. 227 der deutschen, p. 284 der französischen zweiten Auflage.

${ }^{12}$ De CANDolle spricht sich zurückhaltender aus, aber der Sinn ist der erwähnte und wurde so in fast allen neueren Untersuchungen über GaLToN bei aller hoher Anerkennung seines Werkes formuliert.

${ }^{13}$ Diese Bezeichnung stammt nicht von de Candolle.

${ }^{14}$ Bei der Vergleichung der aus den einzelnen Völkern stammenden Anzahl von bedeutenden Gelehrten - nach dem Urteil der angesehensten Akademien - hat er dann freilich im ausgiebigsten Maße von statistischen Tabellen Gebrauch gemacht (S. $301 \mathrm{ff}$.). Diese Statistiken wurden ziemlich allgemein anerkannt und wiesen in der Regel jene Tatsachen zahlenmäßig nach, die man vorher qualitativ behauptet hatte. Es kann aber kein Zweifel sein, daß dE CANDolle trotzdem die Ursachen, die zur Wahl eines Gelehrten in eine 
Beschreibung der einzelnen Ursachen oder, wie man besser sagen würde, der «historischen Kräfte»15. Den Begriff der «Ursachen» oder «conditions» bestimmt er nicht näher, sondern verwendet ihn nach dem damaligen Sprachgebrauch; spricht zudem in den Überschriften vor allem von «Influence».

Unter den besonderen Einflüssen untersucht DE CANDOLLE nacheinander jene der Erblichkeit, spezieller Neigungen, der Erziehung, der Religion, der Familientradition, der öffentlichen Meinung, des Staates, der wissenschaftlichen Gesellschaften, der Größe des Landes, der Sprache, der geographischen Lage, des Klimas und der Rasse.

Aus der gruppenhaften und der persönlichen Untersuchung von Forschern beweist DE CANDolle, da $\beta$ die Vererbung nur eine sehr allgemeine Rolle spielen kann. Die vorhandenen «Tatsachen unterstützen die Annahme einer Erblichkeit nach großen Kategorien der Fähigkeiten viel mehr, als die einer besonderen Fähigkeit». ${ }^{16}$ Um dann daraus große wissenschaftliche Werke hervorgehen zu lassen, sind Charaktereigenschaften entscheidend: Willensstärke, Ausdauer, Wißbegierde, Tatkraft, Ordnungssinn,

Akademie führten, nicht umfassend genug untersuchte; vor allem jene Bedingung nicht, die dazu führen, daß ein Gelehrter nicht in eine große Akademie als ausländisches Mitglied aufgenommen wird. Meines Wissens waren z.B. Gregor Mendel wie JacoB BURCKHARDT nie zu solchen Ehrungen gekommen, obgleich ihre hervorragende Bedeutung unbestritten ist; diese Beispiele konnte natürlich C. nicht kennen.

15 Auf die Frage, wie weit Statistik in der menschlichen Geschichte anwendbar ist, kam DE CANDolle in seinem III. Abschnitt zu sprechen, insbesonders im Paragraphen: «Einwendungen, welche man gegen die auf moralische und soziale Tatsachen angewendete statistische Methode erhoben hat.» Auch diese Ausführungen sind bemerkenswert. Sie knüpfen zur Hauptsache an QuETELET an. Das großartige IV. Kapitel von ЈАКов BERNoullis Wahrscheinlichkeitsrechnung kannte er anscheinend nicht, und somit auch nicht dessen Begründung des «Gesetzes der großen Zahl» im entscheidenden ursprünglichen Zusammenhang, sowie Bernoullis weitere Betrachtungen. - Den Kern des Problemes jedenfalls sieht er nicht, nämlich, daß a) die in der menschlichen Geschichte wirkenden Kräfte außerordentlich zahlreich sind und neben die Natur- die Sozialkräfte treten; und $b$ ) daß die meisten historischen Erscheinungen Resultanten verschiedener historischer Kräfte entsprechen, so daß das einmalige, mehrmalige oder sehr komplexe Auftreten der durch die historischen Kräfte bewirkten Erscheinungen die Regel ist. Nur bei sehr allgemeinen Phänomen, wie sie vor allem die Demographie bietet, treten die gleichen Erscheinungen, durch die gleichen Ursachen bedingt, in genügend großer Zahl auf, um Statistiken zu ermöglichen oder Korrelationen zahlenmäßig nachzuweisen. Diese Grundtatsachen des geschichtlichen Lebens einmal eingehend zu erörtern, liegt außerhalb der Möglichkeit dieses Aufsatzes.

16 S. 243. 
Beobachtungsgabe - und typisch für die Epoche, in welcher die Verachtung der Philosophie vorherrschte - das Vermeiden metaphysischer Spekulation, klares Urteil, gutes Gedächtnis, Aufmerksamkeit, Ideenfülle usf. Von nachweisbarer Wirkung sind auch besondere Neigungen. Aber es ist im Einzelfall nicht oder schwer zu entscheiden, ob sie angeboren (also vererbt), durch starke Jugendeindrücke bestimmt oder durch ein Wegfallen anderer, sonst üblicher Umstände erzeugt sind. Aber auch solche Bedingungen genügen noch nicht für sich, um die große wissenschaftliche Persönlichkeit hervorzubringen: diese ist noch abhängig von der Umwelt, der geschichtlichen Lage.

Hier gibt es vor allem einen Faktor, der selbst die größten Potenzen vernichtet: die Ansicht, welche die Wissenschaft als abgeschlossen darstellt ${ }^{17}$ und ein Individuum in den Bann schlägt.

Der Einfluß der Schulen ist nach DE Gandolle, der den Hauptteil seiner Bildung dem Elternhaus und Selbststudium verdankte, problematisch. «Man muß zugestehen: Berühmte Gelehrte geben guten Unterricht, aber guter Unterricht macht nicht berühmte Gelehrte», ${ }^{18}$ und er fügt noch an: «Eine bedauernswerte Wirkung des Unterrichtes besteht darin, daß er die Originalität vermindert. Es ist unmöglich, fortwährend dem Unterricht zu folgen, viel zu lesen, kurz zu lernen, ohne ein wenig von der Ursprünglichkeit der Ideen einzubüßen, welche den originellen Geistern eigen ist.» Er findet sich darin in Übereinstimmung mit EULER, der auch vor zu umfassender oder ständiger Lektüre warnte ${ }^{19}$. Aber man wird doch zugeben müssen, daß, wie die Folgezeit bewies, die Urteile DE CANdolles über die allgemeine Schulung einseitig waren: aus der Abneigung eines sich zum Stadtpatriziat zählenden Mannes gegen die allgemeine Volksschule entsprangen. Zudem beachtete er Rhythmus von schöpferischer Muße und wissenschaftlicher Rezeption ungenügend. Er selbst baute seine Untersuchungen auf ein umfassendes literarisches Fundament auf. Aber er mied Übersättigung und blieb seiner großen Konzeption treu.

Im Kapitel «Der Einfluß der Religion» würdigt er die hohe Bedeutung des Christentums für die Wissenschaft.

«Die nichtchristlichen Länder stehen der wissenschaftlichen Bewegung

17 S. 259.

18 S. 260.

${ }^{19}$ Vgl. Eduard Fueter, Geschichte der exakten Wissenschaften in der schweizerischen Aufklärung (1680-1780), Aarau 1941, S. $117 \mathrm{f}$. 
völlig fern ${ }^{20}$. Man muß jedoch daraus nicht schließen, daß man erst Christ werden muß, um ein ausgezeichneter Gelehrter zu werden, denn viele Beispiele widersprechen dieser Annahme. Man kann nur sagen, daß die christliche Religion die Entwicklung der Wissenschaften durch ihren allgemeinen Einfluß auf die Zivilisation begünstigt hat. Man kann wenigstens mit Sicherheit sagen, daß sie im modernen Zeitalter die einzige Religion ist, die mit einer ernsthaften wissenschaftlichen Entwicklung zusammengeht.»

Insbesonders der Dogmengeschichte spricht er weitgehend jede Bedeutung ab. Denn man wisse nie, wie weit kirchliche Lehrsätze tatsächlich befolgt wurden. Es kann kein Zweifel sein, daß hier nun die spezifischen Schwächen in der Beurteilung de Candolles beginnen. Auch wenn bis heute die Religions- und Geistesgeschichte viele Fragen offen gelassen hat und selbst eine Schrift wie etwa Ernst Troeltsch' Die Bedeutung des Protestantismus für die Entstehung der modernen Welt (1906, 5. Auflage 1928) oft mehr eine These großartig vortrug, als eine wissenschaftliche Wahrheit begründete, so haben Dogmen tiefen Einfluß ausgeübt ${ }^{21}$. Man steht vor der Tatsache, daß besonders die protestantischen Nationen und - was noch viel charakteristischer ist - die protestantischen Stände in der Eidgenossenschaft oder gewisse protestantische Gebiete Süddeutschlands seit der Gegenreformation fruchtbarer waren für die moderne Forschung als andersgesinnte Staaten und Landschaften. Dafür gilt es die Ursachen in gründlicherer Art zu suchen, als die aphoristischen Betrachtungen DE CANDOLles tun. Dagegen hat DE CANDOLLE wieder recht, wenn er implizit vor einer Überschätzung der Dogmengeschichte warnt. Die letzten Jahrzehnte historischer Untersuchungen haben gezeigt, wie vielseitig und verwickelt die Zusammenhänge sein können. So sind die relative Freiheit, die «Priesterehe», ${ }^{22}$ d. h. das protestantische Pfarrhaus, der Mangel einer kirchli-

${ }^{20}$ Diese Anschauung ist typisch für die früheren Epochen europäischer Geschichtbetrachtung. Sie ist natürlich nicht mehr haltbar. Über die Vielseitigkeit wissenschaftlicher Bemühungen etwa außerhalb des christlichen Kulturkreises im 14. Jahrhundert orientiert vorzüglich und in enzyklopädischer Weise Georg SARTon, Introduction to the History of Science, vol. III, Baltimore 1948/49.

21 Über die Auseinandersetzungen zwischen Orthodoxie, protestantischer Neuscholastik einerseits, Cartesianismus und Kopernikanismus vgl. das unter ${ }^{19}$ angegebene Werk, S. 14.ff., zudem Josef Bohatec, Die cartesianische Scholastik in der Philosophie und reformierten Dogmatik des 17. Jahrhunderts, I. Teil, Leipzig 1912. - Die grundsätzlichen Probleme hat in lichtvoller Klarheit und Überzeugung EMIL Brunner in Offenbarung und Vernunft (Zürich 1941) herausgestellt.

${ }^{22}$ Über diese Wirkung hat DE CANDOLLE wohl die bemerkenswertesten Feststellungen ge- 
chen Hierarchie, das Nachwirken des Humanismus im calvinistisch-zwinglianischen Bereich, die Komponenten der «natürlichen Religion» usf. von unterschiedlicher, aber nachweisbarer Bedeutung gewesen. Aber sie standen in Wechselwirkung zu wirtschaftlichen und militärischen Voraussetzungen. Zudem war überaus wichtig, daß die Entdeckung Amerikas vor allem den romfernen Nationen zugute kam, die aus nationalen Gründen dem römisch-katholischen Episkopat widerstrebten. $\mathrm{Daß}$ für sich allein auch dieser Faktor nicht überschätzt werden darf, ergibt sich aus der Tatsache, daß der Silberstrom aus den Minen von Potosi in erster Linie der romtreuen, spanischen Krone große Mittel spendete.

\section{iV. Alphonse de Candolle und Jacob Burckhardt}

\section{Allgemeine Anschauungen}

An historischer Tiefe übertrifft das Werk «Weltgeschichtliche Betrachtungen» von BURGKHARDT, das fast gegen seinen Willen erschien, DE CANDoLles Buch natürlich sehr. Auch ist des Baslers Blickfeld universell, nach Epochen, Persönlichkeiten, Berufen und Berufungen; während sich der Genfer bewußt beschränkte.

troffen: «Die Wissenschaften wären nicht so weit fortgeschritten, wie sie es heute sind, wenn Linné, Hartsöcker, Euler, Jenner, Wollaston, Olbers, Blumenbach, Robert Brown, Berzelius, Encke, Mitscherlich, Agassiz usw. nicht geboren worden wären. Glücklicherweise waren ihre Väter, obwohl Geistliche, nicht dem Zölibat unterworfen. Nehmen wir aus der Liste der Gelehrten der protestantischen Länder die Söhne der Pastoren heraus, so ist das Gleichgewicht zwischen der Bevölkerung der beiden Bekenntnisse bezüglich ihrer Beeinflussung der Wissenschaften fast hergestellt» (S.266). Wenn aber dann DE CANDOLLE weiterfährt: «So hat eine reine Disziplinarregel, die den Dogmen ganz fern stand und die in der römischen Kirche keineswegs von jeher existiert hat, in den katholischen Ländern für die Wissenschaften die schlimmsten Folgen gehabt», so ist dieser Sachverhalt nun viel zu einfach dargestellt. Zudem wäre zu erörtern, warum gerade die mittelalterlichen Mönche in Europa die Förderer der Wissenschaften - wie die Mauriner im 17.Jahrhundert für die geschichtlichen Hilfswissenschaften - waren: immer kommt es auf die geschichtliche Umwelt und weitere Faktoren an. Das Zölibat selbst entscheidet so wenig wie das protestantische Pfarrhaus für sich betrachtet: daß dieses mit relativ hoher individueller Bildung oder wissenschaftlicher Neigung einherging und die freie gelehrte Tätigkeit achtete, sowie die Förderung der Forschung im Kulturkreis beruflicher Tätigkeit würdig schien, usf., hatte den historisch belegten Erfolg. - Bei den Maurinern war die Veranlassung auch klar: im literarischen Kampf der Konfessionen kam es auf die möglichst stichhaltige historische Dokumentation an, auf die Ausbildung der Diplomatik; wie früher einst auf die bessern Methoden der protestantischen Zenturiatoren im 16.Jahrhundert (vgl. EDUARD Fueter sen., Geschichte der neueren Historiographie, 3. Auflage 1936, S. $24.9 \mathrm{ff}$., $309 \mathrm{ff}$.). 
Noch wesentlicher sind persönliche Momente. JacoB BuRcKHARDT ging von einer skeptischen Warte aus. Er stand unter dem «Eindruck von der Hinfälligkeit und Unsicherheit alles Irdischen» ${ }^{23}$; wogegen DE CANDoLle sowohl den Optimismus besaß, die Voraussetzungen für eine immer bessere Pflege der Forschung durch seine Untersuchungen schaffen zu wollen, als auch begründete, weshalb die wissenschaftliche Kultur der weißen Rasse in der Moderne kaum mehr untergehen könne ${ }^{24}$. BURGKHARDT ${ }^{25}$ sieht die furchtbaren Gefährdungen Europas voraus, DE CANDolde die Steigerung der Forschung und sogar der äußern Kultur im allgemeinen.

Beinahe entgegengesetzt ist ihre Methode. Beide waren zwar scharfe Beobachter und verlangten diese, aber in völlig verschiedener Art. Während DE GANDOLLE seine Untersuchungen anstellte, um naturwissenschaftlich genau oder sogar statistisch erfaßbar zu wissen, welche «Wachstumsbedingungen» die «Humanbotanik» benötige, so daß die «Rosaceen der Gelehrten» prächtig erblühen, so steht bei BURCKHARDT immer die Mahnung, «das sich Wiederholende, Konstante, Typische»unddas «Interessante» durch stete Lektüre herauszugreifen, vor allem sich der Gefährdung aller Individualität zu erinnern: welches Trümmerfeld nicht gereifter Talente und Genies die Geschichte der Menschheit aufweise. Dadurch ist BurckHARDT auch äußerst sensibel gegenüber den kleinen Kräfteverschiebungen, welche alles zunichte machen können, und er legt der Natur teleologische Ziele bei, wenn er etwa ausspricht: «Die Natur verfährt dabei (in der Hervorbringung großer Persönlichkeiten) mit ihrer bekannten Sparsamkeit, und das Leben bedroht die Größe von Jugend auf mit ganz besonderen Gefahren, darunter die falschen, d.h. die der wahren Bestimmung des großen Individuums im Widerspruch stehenden Richtungen, welche vielleicht nur eben ein Minimum zu stark zu sein brauchen, um unüberwindlich zu sein.» ${ }^{26}$

Aber trotz aller Spannungen ihrer Überzeugungen und Methoden sind sich de Candolle und Burckhardt darin einig, daß es die Erfüllung vieler Voraussetzungen bedarf, bis ein Talent zur Reife und noch mehr zur Auswirkung gelangt. Auffällig ist nun freilich und überaus bemerkenswert,

${ }^{23}$ Jacoв Burckhardt, Gesamtausgabe, Erster Band, S. VII. - Dazu die ausgezeichnete, «monumentale» Biographie von WeRner KAEGI, Bd. I, S. $197 \mathrm{ff}$.

${ }^{24} \mathrm{~S} .141 \mathrm{ff}$.

${ }^{25}$ Vgl. vor allem die Historischen Fragmente aus dem Nachlaß, 7.Bd.

${ }^{26}$ Burckhardt, Gesamtausgabe, 7. Bd., S. 163. 
daß es für den Naturforscher dE CAndolle keine Geheimnisse darin gibt ${ }^{27}$, sondern nur rational bestimmbare Ursachen und Kräfte (auch wenn sie teilweise noch unbekannt sein mögen), während für den Historiker BURCKHARDT doch eine dem Menschen nicht mehr gegebene Erkenntnis dieses tiefe Geheimnis enthüllen würde : «Die wirkliche Größe ist ein Mysterium.» ${ }^{28}$

Allerdings bezieht sich diese Äußerung in erster Linie auf Dichter und (andere) Künstler, die bei Burckhardt zusammen mit den Philosophen die Naturforscher oder Entdecker an kultureller Bedeutung weit übertreffen, weil sie unersetzlich sind: «Mit den großen Philosophen erst beginnt das Gebiet der eigentlichen Größe, der Einzigkeit und Unersetzlichkeit, der abnormen Kraft und der Beziehung auf das Allgemeine.» Diese Grundeinstellung bewirkt, daß DE CANDOLLE und BuRGKHARDT manchmal inkommensurabel sind: die Forscher krönt «weder die absolute Fähigkeit noch das sittliche Verdienst und die Hingebung an die Sache - denn diese verleiht Würde, aber nicht Größe -, sondern die großen Entdecker in bestimmten Richtungen, nämlich die Auffinder von Lebensgesetzen ersten Ranges» ${ }^{29}$. So behauptet BurcKhardT die relative, fachliche Größe der Forscher, während für DE CANDOLLE darin gerade die überragende Fähigkeit liegt. Nach BURGKHARDT sind die großen Entdecker zwar individuell unauswechselbar - sofern sie sich in die Reihe der Philosophen begeben wie Galilei, KePLer und wenige andere; in ihren Leistungen aber ersetzbar; ähnlich wie sich die Entbehrlichkeit von Columbus behaupten ließe. Er zitiert darin K. E. voN BAER: «Amerika würde bald entdeckt worden sein, auch wenn Columbus in der Wiege gestorben wäre», ${ }^{30}$ was man von Aeschylus, Phidias, Plato, Raffael keinesfalls sagen könne.

Aus de Candolles Betrachtungen leiten sich andere Schlüsse ab. Seine Grundansicht darf man wohl dahin zusammenfassen, daß bei gleichbleibenden günstigen kulturellen Voraussetzungen die Menschheit oder ein Volk genügend Begabungen erzeuge, um auf die eine oder andere Weise große Talente in den Wissenschaften hervorzubringen, die sich dann für den Fortschritt der wissenschaftlichen Forschung einsetzen. Aus der Kon-

27 «Die Anzahl und Beschaffenheit der kennzeichnenden Charaktere, seien sie ererbt, durch die unbekannten Ursachen der Variation bewirkt oder nach der Geburt durch äußere Faktoren bestimmt, bestimmen die Anpassung jedes einzelnen Individuums an die Umwelt, in der es sich befindet» (XIV).

${ }^{28}$ BurckhardT, a.a.O., S.161.

${ }^{29}$ Ib. S. $165 / 6$.

${ }^{30} \mathrm{Ib}$. S. 165. 
tinuität der günstigen Bedingungen und der vorhandenen Begabungen ergibt sich also die Kontinuität des Fortschritts der Erkenntnis ${ }^{31}$, wobei nicht deren Originalität, sondern deren objektive Wahrheit entscheidend ist $^{32}$. Dafür braucht es eine große «sittliche Kraft» : «Man kann nicht die Erfordernisse der absoluten Wahrheit zu seinem täglichen Gebrauch machen, ohne dazu gewohnheitsmäßig geführt zu werden, auch außerhalb der Wissenschaft das Wahre vorzuziehen und aufrechtzuerhalten»». ${ }^{33}$

Mit diesem sittlichen Imperativ ist die Frage nach der Freiheit des Menschen zwingend gestellt. Es ist überaus charakteristisch, daß sich BuRcKHARDT darüber nur sehr zurückhaltend äußert und daß DE CANDOLLE im Zusammenhang mit Quetelets Physique sociale (1869) darauf zu sprechen kommt. QUeTELET hatte aus seinen sozialstatistischen Untersuchungen gefolgert: «Die Willensfreiheit der Menschen verschwindet und bleibt ohne nachweisbare Wirkung, wenn sich die Beobachtung über eine große Anzahl von Individuen erstreckt». ${ }^{34}$ DE CANDolle gebührt das Verdienst, daß er seinerseits auf die Fehlschüsse von QueTelet hinwies und erklärte, daß das Problem der Willensfreiheit des einzelnen Individuums mit den vorgebrachten Zahlen nichts zu tun habe. So stimmen denn BurcKHardT und DE Candolle wieder darin überein, daß außer den biologischen (Erb-) Faktoren und der sozialen oder historischen Umwelt der durch den freien Willen teilweise oder weitgehend lenkbare Charakter, sowie die überlegte Auswahl der Forschungen entscheidend sind. Obgleich sich bei DE CANDoLle stellenweise eine Unsicherheit - unter dem Einfluß von Galton über die Vererbbarkeit des starken Willens geltend macht und er zudem eine sehr differenzierte Anschauung über die Auswirkungen «guter Eigen-

31 «Der Mann, der von seinen Eltern oder Vorfahren eine gewisse Dosis Fleiß, Aufmerksamkeit, Urteilskraft, Wille, Wißbegierde, Mut, Ordnungssinn, Neigung zur Beobachtung und zur Unabhängigkeit des Geistes und diese in einer glücklichen Kombination erhalten hat, kann in allen ernsten, literarischen, wissenschaftlichen, juristischen und Verwaltungsarbeiten und im allgemeinen in Angelegenheiten, welche Ehrlichkeit und Fähigkeit fordern, Erfolg haben. Wenn er durch günstige Umstände in der Familie, im Vermögen, in der Erziehung oder in andern Dingen unterstützt wird, wenn die öffentliche Meinung und die bestehenden Einrichtungen ihm günstig sind, wenn er nicht durch übermäßige Phantasie von vernünftigen Wegen abgèbracht wird, wenn er nicht durch gewisse Laster und Fehler, die einem arbeitsamen Leben und der Forschung nach der Wahrheit hinderlich sind, aufgehalten wird, kann er es sehr weit bringen.»

${ }^{32}$ Vgl. S. 10/1, 112/3.

${ }^{33} \mathrm{~S} .113$.

34 S. 15. 
schaften» vorträgt, so faßt er diese Tatsache doch dahin zusammen: «Indessen gibt es Vorzüge, die vorteilhaft oder notwendig für jede Art des Erfolges sind. Hieher gehört: der Wille, der entweder stark oder dauernd sein muß; der gesunde Menschenverstand, der Tätigkeitstrieb, in Hinblick auf welchen Voltaire erklärte: ,Die Faulen sind in allen Berufen Mittelmäßige'.» In gleicher Art, aber in seiner wunderbaren, ehrfurchtsvollen Sprache stellt Burckнard fest: «Dabei ist ihm (dem großen Menschen) eigen und natürlich die Fähigkeit, sich nach Belieben auf eine Sache zu konzentrieren ... .» In ihm «lebt vor allem wirklicher Wille, sich der Lage zu bemächtigen, und zugleich eine abnorme Willenskraft»s. Es ist die Seelenstärke, "welche es allein vermag und daher auch allein liebt, im Sturme zu fahren» ${ }^{35}$.

Auffallend ist dabei allerdings wieder, wie sowohl BURckнardt wie DE Candolle die den Willen solcher Persönlichkeiten hemmenden Faktoren: geringe natürliche «Vitalität» ${ }^{36}$ und Krankheiten, nur nebenbei erwähnen, obgleich es eine lange und tragische Geschichte menschlichen Versagens in dieser Hinsicht gibt. Allerdings scheint die Wissenschaftsgeschichte vom Einfluß der medizinischen Bedingungen auf die großer Forscher noch nicht geschrieben. Dabei steht freilich zu vermuten, daß weniger die einzelnen Erkrankungen wesentlich sind, als der Grad an Widerstandsfähigkeit oder «Vitalität», ${ }^{34}$ welcher fortbesteht oder geschwächt wird. Was wäre etwa aus Euler geworden, wenn sein Augenstar und die damit verbundenen Krisen ${ }^{37}$ ein allgemeines organisches Leiden nach sich gezogen hätten? Da aber seine Lebens- und Willenskraft ungebrochen blieben, so wurde seine Erblindung kein Hindernis, ja es war ihm vergönnt, das berühmteste und

${ }^{35}$ BUrckhardt, a. a. O., S. 180. - Aus dem Zusammenhang ergibt sich, daß BURCKHARDT zwar an jedes große Individuum denkt, aber in erster Linie an Staatenlenker.

${ }^{38}$ Die Vorstellung einer «Lebenskraft» lehnt DE CANDolle ab (im Kapitel: «Die Transformation der Bewegung bei den organischen Wesen»). Aber es zeigt sich, daß er daraus auch etwas anderes versteht als «Vitalität», d.h. einen Sammelbegriff für vornehmlich erblich bedingte Eigenschaften, welche die physische Lebenstüchtigkeit günstig beeinflussen. Dazu würde somit auch die oft für gelehrte Leistungen so wichtige Lebensdauer zählen. Bei den Naturforschern und Medizinern des 17. und 18. Jahrhunderts in der Schweiz fällt auf, daß sie gegenüber der für jene Epoche angenommenen durchschnittlichen individuellen Lebensdauer lange lebten: z. B. Johannes I Bernoulli 80 Jahre, Daniel I Bernoulli 82 Jahre, Johann Jakob Scheuchzer 62 Jahre, Albrecht von Haller 69 Jahre, Charles Bonnet 72 Jahre, usf.

${ }^{87}$ Vgl. R. Fueter im Beginn seines Aufsatzes Über eine Eulersche Beweismethode in der Zahlentheorie, Schweiz. med. W sch. 69, Nr. 43, S. $103 \mathrm{ff}$. 
bedeutendste Beispiel eines «Altersgenies» zu werden ${ }^{38}$. Der fast völlige Mangel an Senilität des über Siebzigjährigen setzt immer wieder in Erstaunen: die Arbeiten seines hohen Alters sind kaum weniger originell als diejenigen des Mannesalters. Auch bei Johannes vom Müller - um einen bekannten Geschichtschreiber noch herauszugreifen - steht man unter dem Eindruck einer ähnlich überdurchschnittlichen Vitalität. Er überwand viele und schwere Schicksalsschläge trotz seelischer Verzweiflungsphasen erstaunlich gut $^{39}$.

Mit diesen Andeutungen über «Bedingungen wissenschaftlicher Größe in der europäischen Kultur» ist das Problem natürlich nur gestreift, wie auch jenes der umfassenden kritischen Würdigung oder gar Fortführung des Werkes von Alphonse de Candolle. Er selbst hat stets darauf hingewiesen, wie ergänzungsbedürftig seine Schrift noch sei, und zudem Aspekte behandelt - wie über die geographische Verbreitung der Wissenschaften, über nationale Eitelkeit und wissenschaftliche Berühmtheiten, das Problem der wissenschaftlichen Sprache usf. -, die hier übergangen wurden. Entscheidende Probleme hat er ungenügend oder gar nicht bemerkt, und sie wurden auch hier nicht berücksichtigt. So wäre vor allem auf den allgemeinen Impuls, der von neuen wissenschaftlichen Vorstellungen ausgeht - wie etwa der von Henry E. Sigerist so überzeugend nachgewiesene Einfluß des «anatomischen Gedankens» auf eine große Zahl von Medizinern, die damit zu originellen Leistungen befähigt wurden -; auf den Einsatz neuer Instrumente (Fernrohr, Mikroskop usf.); auf neue Methoden (Infinitensimalrechnung, Wahrscheinlichkeitslehre; Experiment, Sektion; team-work, cooperative research usf.) hinzuweisen, welche große Entdeckungen selbst einem oft eher mittelmäßigen Talent ermöglichte, wenn es nur fleißig war, so daß von einem bestimmten Zeitpunkt an der Grad der Anstrengung oder der Begabung absinken kann. Natürlich gab auch DE CANDOLLE stets zu bedenken, daß günstige Umstände individuell noch keine großen Forscher schufen, sondern nur befördern.

${ }^{38}$ Vgl. die vorzügliche Darstellung von Andreas Speiser, Die Basler Mathematiker, Neujahrsblatt, Basel 1939, S. 46f. - Nicolaus Fuss sagt in seiner Lobrede allgemein von Euler «Herr Euler war von einer gesunden und dauerhaften Leibesbeschaffenheit. Ohne diese würd er schwerlich so viele Erschütterungen haben widerstehen können, mit denen die Heftigkeit und Menge seiner Krankheiten seinen Körper bestürmt hatten» (Opera I, 1, S. XC).

${ }^{39}$ Vgl. die leider unvollendet gebliebene Biographie von KarL Henking, Johannes von Müller, 2 Bände, Stuttgart und Berlin 1911, 1928, bes. II, S. $546 \mathrm{ff}$. 
Die Bedingungen aber allgemein zu erörtern, die als günstige Ursachen angesprochen werden dürfen, ist immer ein ernsthaftes Ziel.

Es ist heute aktueller als je. Dabei stehen die finanziellen Probleme als die dringlichsten meist voran. Aber es sind nicht die einzigen.

Daher mögen zum Schluß dieser Erinnerungen an DE Candolle die folgenden zwanzig «Gebiete» für die Blüte der Wissenschaften zitiert werden, welche jener unter dem Titel «Günstige Ursachen» als eine Art geistigen Vermächtnisses seinem Werk im Fettdruck einfügte ${ }^{40}$ :

\section{«Günstige Ursachen}

1. Ein bedeutender Prozentsatz von Leuten, die zur reichen und wohlhabenden Bevölkerung gehören, im Verhältnis zu denen, die beständig arbeiten müssen, um leben zu können, und besonders zu denen, die Handarbeit leisten müssen.

2. Ein erheblicher Prozentsatz von Individuen in den reichen und wohlhabenden Klassen, die mit ihren Einkommen zufrieden sind, ein leicht zu verwaltendes Vermögen haben und infolgedessen geneigt sind, sich mit geistigen, wenig oder gar nichts einbringenden Dingen zu befassen.

3. Alte Geistes- und Gefühlskultur, die seit mehreren Generationen auf wirkliche Dinge und auf wahre Gedanken gerichtet ist (Einfluß der Erblichkeit).

4. Einwanderung von fremden, gebildeten und rechtschaffenen Familien, die Interesse für wenig oder gar nichts einbringende geistige Arbeiten haben.

5. Das Vorhandensein von Familien, die für die Wissenschaften und für geistige Beschäftigungen jeglicher Art günstige Traditionen haben.

6. Gut organisierter elementarer und besonders mittlerer und höherer Unterricht, der unabhängig von den politischen und religiösen Parteien darauf gerichtet ist, die Forschung anzuregen und solche junge Leute und Professoren zu begünstigen, die den Wissenschaften ergeben sind.

7. Reichliche und gut organisierte Mittel für die verschiedenen wissenschaftlichen Arbeiten (Bibliotheken, Observatorien, Laboratorien, Sammlungen).

${ }^{40} \mathrm{~S} .326 \mathrm{ff}$. 
8. Ein für das Wahre und Wirkliche mehr als für Dichtung und Phantasie interessiertes Publikum.

9. Die Freiheit, jede Ansicht, wenigstens über wissenschaftliche Gegensätze, zu äußern und zu veröffentlichen, ohne einigermaßen erhebliche Unannehmlichkeiten erleiden zu müssen.

10. Eine öffentliche Meinung, die den Wissenschaften und denen, die sich damit beschäftigen, günstig ist.

11. Eine Freiheit, die gestattet, jeden Beruf auszuüben, keinen auszuüben, zu reisen und alle persönlichen Dienste zu meiden außer freiwillig übernommenen.

12. Eine Religion, die wenig von dem Autoritätsprinzip Gebrauch macht.

13. Eine Geistlichkeit, die unter ihren Mitgliedern und in der Öffentlichkeit den Unterricht fördert.

14. Eine Geistlichkeit, die nicht zum Zölibat gewungen ist.

15. Gewohnheitsmäßiger Gebrauch einer der drei wichtigsten Sprachen: englisch, deutsch, französisch. Genügend verbreitete Kenntnis dieser Sprachen in den gebildeten Klassen.

16. Kleine unabhängige Länder oder Föderation kleiner unabhängiger Länder.

17. Geographische Lage unter einem gemäßigten oder nördlichen Klima.

18. Nachbarschaft zivilisierter Länder.

19. Zahlreiche Akademien oder wissenschaftliche Gesellschaften.

20. Gewohnheit zu reisen und besonders sich in fremden Ländern aufzuhalten.» 